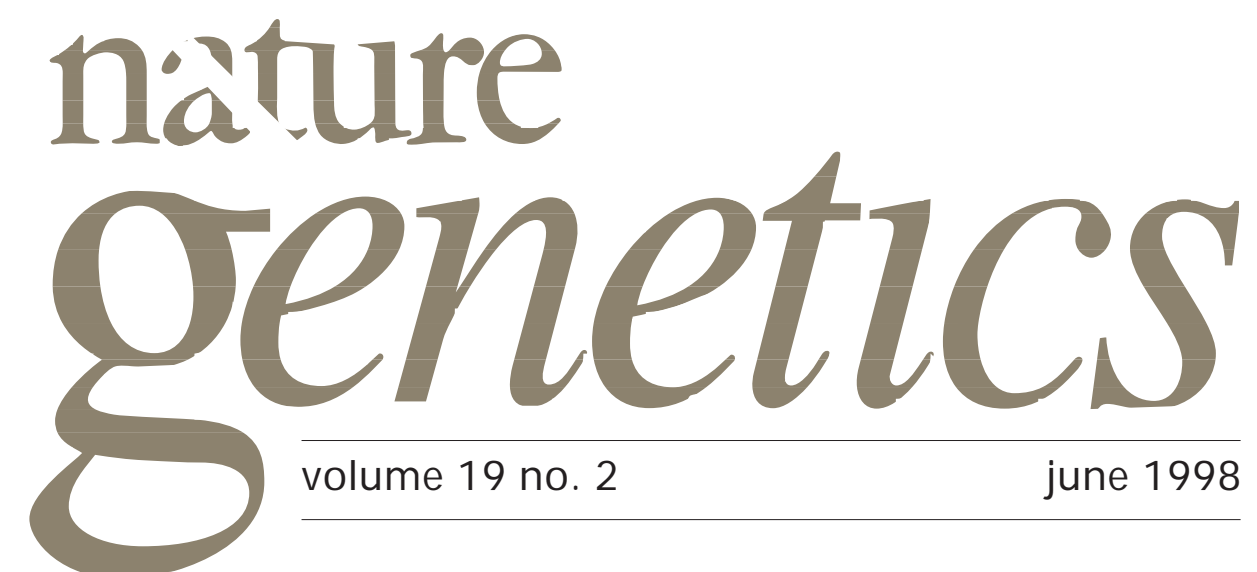

\title{
Be fruitful and immortalize
}

The oldest human, Jeanne Calment—who died last year at 122 years and 164 days - had forebears who lived to an exceptionally old age ${ }^{1}$. Recently, Tom Perls and colleagues, in a population-based study, found that many centenarians have long-lived siblings ${ }^{2}$. These observations, and others, have implicated a major hereditary influence on longevity. A recent meeting on the Genetics of Aging* - as well as several papers in this issue-shed new light on the many genetic factors and cellular processes that contribute to ageing.

Normal mitotic cells are 'mortal' in culture and 'senesce' after a finite number of cell divisions. Telomere shortening has proved to be a potent molecular trigger of cellular senescence; the introduction of telomerase into several cell types enables them to avoid cellular senescence and extends their lifespan ${ }^{3}$. Recent data also indicate that telomerase is critical for maintaining some highly proliferative organs that undergo continual cell renewal from a reservoir of stem cells-lategeneration mice deficient in telomerase have severely compromised proliferative systems, including diminished haematopoietic colony formation and defective spermatogenesis ${ }^{4}$. Consistent with this, Yoichi Shinkai and co-workers report in this issue that undifferentiated embryonic stem cells lacking telomerase ultimately lose the capacity to proliferate. With telomerase's influence on cellular mortality evident, efforts are now focused on identifying the factors that regulate its activity in mammalian cells. One such potential factor is described by Benoit Chabot and colleagues on page 199; they demonstrate that UP1, the proteolytic fragment of heterogeneous nuclear riboprotein A1, binds specifically to telomeric repeats and associates with telomerase, suggesting that UP1 might be directly involved in telomere biogenesis.

Although telomerase is critical to the replicative capacity of normal cells, it also appears to line the path leading to cellular immortalization in tumorigenesis. Increased levels of telomerase are detected in cancerous tissues and transformed cells, and some studies suggest that its activation occurs late in tumour progression. On page 182, William Gerald and co-workers show that telomerase expression is detected at very early stages in breast and colon tumorigenesis and also in some mitotically inactive normal cells, suggesting that, in some cases, the genetic changes that initiate cancer may occur within target cells that already express telomerase.

Although senescent cells accumulate with age, it has yet to be proved that cellular senescence actually causes the aged phenotype. Senescent cells secrete inflammatory cytokines and enzymes capable of degrading extracellular matrix proteins, and it is plausible that even a relatively small number of senescent cells could have a destructive impact on tissue integrity and function. Several presenta- 
tions at the meeting described efforts to understand the altered state of senescent cells and their influence on ageing. For example, Walter Funk and colleagues at Geron Corporation are using DNA chip technology to establish a comprehensive profile of senescence-associated changes in gene expression, and Judith Campisi and co-workers, at Lawrence Berkeley Laboratory, California, have identified a senescence-responsive promoter element that is activated in replicationexhausted cells. Campisi proposes to link this element to a reporter molecule in transgenic mice to determine precisely where and when senescent cells occur in the context of the whole organism. Linking this element to a toxic reporter, which would kill cells that express it, may determine whether mice that fail to accumulate senescent cells have mitotically competent tissues that retain a greater degree of structural preservation with age.

All aerobic cells require oxygen to survive, but they are also under chronic attack by superoxide radicals generated by oxidative metabolism. These reactive metabolites disrupt the integrity of many cellular macromolecules (including DNA) and oxidative damage is found to increase with ageing. Genes encoding the proteins that protect the cell from oxidative stress — such as superoxide dismutases (SOD) and catalase - may therefore serve as 'longevity-assurance' genes. In support of this hypothesis, Gabrielle Boulianne and colleagues report, on page 171, that overexpression of human SOD1 exclusively in the motor neurons of Drosophila extends lifespan by $40 \%$ compared with wild-type controls.

It has been suggested that the accumulation of DNA damage, arising from oxidative attack and other cellular stresses, contributes to age-related deterioration. High levels of genomic instability are associated with Werner syndrome, a rare human disorder that displays many (but not all) of the manifestations of accelerated ageing. This disorder is caused by a mutant DNA helicase, implicating this protein, and others involved in DNA maintenance and repair, as factors in the ageing process. Youichi Odagiri and colleagues, on page 116, provide evidence that the rate of DNA mutations correlates with lifespan-a mouse strain prone to accelerated senescence was found to have an increased rate of somatic mutation accumulation compared with a corresponding strain that is resistant to accelerated senescence. Additional evidence that genetic factors influence lifespan is reported by Gary Van Zant and colleagues, on page 114, who have mapped a putative gene that causes variability in the lifespan of genetically identical mice.

Studies of other model organisms have provided insight into the genetic mechanisms associated with ageing. Leonard Guarente and colleagues recently demonstrated that ageing in Saccharomyces cerevisiae is associated with the accumulation of extrachromosomal rDNA circles ${ }^{5}$. Guarente believes that this is likely to be a common mechanism underlying ageing in all cells, and is searching for evidence of rDNA circles in aged human cells. The Caenorhabditis elegans larva has two alternate developmental fates, depending on environmental conditions: it can either develop into an adult worm that lives about 20 days, or enter a 'dauer' larval stage, where it can exist for up to six months before re-emerging as an adult. The daf16 gene is one of the genetic gatekeepers controlling entry into dauer phase, and the activity of daf16 in adult worms promotes an extended lifespan. Intriguingly, daf16 is regulated by an insulin-like signalling pathway and it is possible that its human orthologues, FKHR and AFX, may play a role in ageing in $\operatorname{man}^{6,7}$.

As the collective efforts from different research disciplines are unravelling the complex web of genetic factors that influence the rate and way in which individuals age, there is a pressing need to contemplate the future impact of this knowledge. With next year designated 'The International Year of Older Persons' by the United Nations, it is hoped that endeavours to address the concerns of the aged population will also confront the social, health and ethical implications of understanding the genetics of ageing.

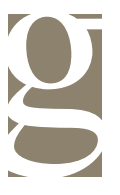

